\title{
Condiciones de trabajo de SIAHO en las universidades públicas de la Costa Oriental del Lago de Maracaibo
}

\author{
SIAHO working conditions in the public universities of the Eastern Coast \\ of Lake Maracaibo
}

\author{
Manuel Borrero \\ borreromanuel80@gmail.com \\ ORCID 0000-0002-8592-2367
}

Universidad del Zulia, Venezuela

Artículo recibido mayo 2019 | Arbitrado en julio 2019 | Publicado en septiembre 2019

\section{RESUMEN}

El estudio buscó identificar las condiciones de trabajo de seguridad industrial, ambiente e higiene ocupacional en las universidades públicas de la Costa Oriental del Lago de Maracaibo. Metodológicamente, la investigación se tipificó como descriptiva, con diseño de campo, no experimental y transeccional. La población estuvo constituida por los trabajadores de mantenimiento y supervisores de SIAHO de las universidades bajo estudio. Para la recolección de datos se utilizó la encuesta mediante un cuestionario, conformado por 18 ítems con escala de frecuencia, validado a través del juicio de expertos y cuya confiabilidad (Alfa de Cronbach), fue de 0,96. El análisis de los datos se realizó mediante la estadística descriptiva, con base en la media aritmética. Se concluye que existe alta presencia para identificar las condiciones de trabajo inseguro, incidente, accidente de trabajo y orden - limpieza como condiciones bajo las cuales se realiza la ejecución de las tareas.

Palabras clave: Accidente de trabajo; seguridad industrial; higiene ocupacional; condiciones de trabajo inseguro; enfermedad ocupacional

\section{ABSTRACT}

The study sought to identify the working conditions of industrial safety, environment and occupational hygiene in the public universities of the Eastern Lake Coast of lake Maracaibo. Methodologically, the research was classified as descriptive, with a field design, not experimental and transectional. The population consisted of industrial safety, environment and occupational hygiene maintenance workers and supervisors from the universities under study. For data collection, the survey was used through a questionnaire, made up of 18 items with a frequency scale, validated through the judgment of experts and whose (Cronbach's alpha) reliability was 0.96 . Data analysis was performed using descriptive statistics, based on the arithmetic mean. It is concluded that there is a high presence to identify unsafe work conditions, incidents, work accidents and order - cleaning as conditions under which the tasks are carried out.

Key words: Industrial accident; industrial safety; occupational hygiene; unsafe working conditions; occupational disease 


\section{INTRODUCCIÓN}

Hoy en día, ha crecido la preocupación por el ambiente laboral y el desarrollo del mismo. Recientemente, las conversaciones no se han hecho esperar en el ámbito mundial, las cuales se han centrado alrededor de las estrategias que se necesitan para manejar los retos de construir sociedades, economías y ambientes más sanos donde el trabajador pueda desenvolverse en un espacio seguro. Es por ello, que se han generado sistemas hacia el medio ambiente laboral, los cuales determinan la importancia concedida al mismo por parte de las empresas, tanto en el caso de que sea abordado tomando el impacto que pueda generar a su grupo de interés, como a su entorno.

De acuerdo con Bárbaro (2010), a nivel mundial, países como Estados Unidos, Alemania y Japón, que cuentan con grandes empresas multinacionales y extienden sus actividades en distintos sectores, tienden a implementar dentro de sus acciones, Sistemas de Gestión Ambiental (SGA) como herramienta para garantizar la uniformidad de sus procesos operativos y la seguridad de los mismos. En estos casos, el desarrollo de su respectivo sistema está acompañado por políticas de comunicación ambiental, las cuales se establecen de manera formal, para darse a conocer a los agentes externos de la compañía a través de su imagen.

En función de lo anterior, dichas acciones generan entre otras posibilidades, que estos sistemas de gestión ambiental puedan actuar como generadores de un efecto catalizador, facilitando la información disponible, promoviendo la comunicación y ayuda desde la gerencia hasta los trabajadores en materia de ambiente laboral. Desde este enfoque, García (2010) indica que los países desarrollados, y en particular sus grandes empresas trasnacionales, presentan una mejor situación de seguridad para sus trabajadores, aunque no están exentas de problemas.

Es por ello, que en las empresas los sistemas de gestión ambiental cada día son más necesarios como elementos de control para minimizar accidentes o pérdidas en las áreas de trabajo. Tal como lo refiere Blanco (2004), las personas causan mayor impacto de accidentes, pero debe recordarse que también son las empresas las que cuentan con los recursos disponibles para ser usados en su protección.

Esta situación en salud y seguridad en el trabajo, fue expresada por la Organización Internacional del Trabajo (OIT) (2005), cuando en un informe expuso que cada año en el mundo 270 millones de asalariados son víctimas de accidentes de trabajo y 160 millones contraen enfermedades profesionales. Partiendo de esta realidad, la Organización Mundial y Panamericana de la Salud (OMPS) (2005), plantea que en América Latina y el Caribe suceden anualmente cerca de cinco por diez accidentes, de los cuales $90 \%$ son mortales, $\mathrm{y}$ se reportan diferentes enfermedades profesionales, aunque existe un gran subregistro por la falta de atención médica a los trabajadores.

En concordancia con lo expuesto, Zúñiga (2005) expresa que las organizaciones públicas y privadas de los diferentes países, no han dado una respuesta razonable a este problema. Por ello, es de suma importancia contar con planes de acción que en la práctica diaria reflejen el compromiso adoptado por la compañía para alcanzar el bienestar y la salud de los trabajadores. 
De este modo, para el investigador, conforme al ambiente actual $\mathrm{y}$ en cumplimiento de este deber, considera que las empresas públicas o privadas, de cualquier sector empresarial, deben realizar la prevención de riesgos laborales mediante la adopción de cuantas medidas sean necesarias para la protección de la seguridad y salud de sus empleados en todos los aspectos. Asimismo, desarrollan como estrategia, sistemas de gestión que parten del nivel gerencial para tratar de una forma sistemática los aspectos referidos con el medio ambiente de trabajo.

Al respecto, explica Fernández (2005, p. 158), "la alta dirección está comprometida directamente con los programas medioambientales mediante procedimientos formalizados y actuaciones informales". Igualmente agrega que el sistema de gestión ambiental, "debe ser capaz de retroalimentar para caminar así hacia la mejora permanente interna $\mathrm{y}$ externa que conduzca a disminuir los accidentes de trabajo".

En atención a la problemática expuesta, las empresas públicas o privadas evidentemente emplean un sistema de gestión en materia de Seguridad Industrial, Ambiente e Higiene Ocupacional (SIAHO); tal y como lo señala VanDer (2010, p. 14), cuyo objetivo fundamental "es la identificación de todos los riesgos asociados con las actividades, la aplicación de procedimientos de trabajo $\mathrm{y}$ acciones preventivas para reducir, controlar o eliminar cada uno de esos riesgos presentes en una actividad".

En torno a ese aspecto, la Comunidad Andina de Naciones (2005), el 26 de septiembre del 2005 hace explícitos los elementos importantes de la gestión de Seguridad Industrial, Ambiente, Ambiente e
Higiene (SIAHO), los cuales pueden visualizarse claramente a través del instrumento Andino de Seguridad y Salud en el Trabajo (propuesto por el reglamento, Resolución 957), vinculante para los cinco países de la subregión Andina: Venezuela, Colombia, Ecuador, Perú y Bolivia. En su artículo 1 recomienda que los países miembros lo desarrollen teniendo en cuenta los siguientes aspectos: gestión administrativa, técnica, del talento humano, y procesos operativos básicos.

En el caso de Venezuela, con la entrada en vigencia de la Ley Orgánica de Prevención, Condiciones y Medio Ambiente de Trabajo (LOPCYMAT, 2005), surgen en la legislación laboral venezolana componentes de suma importancia, dado que esta ley tiene como propósito establecer normas y lineamientos que permitan garantizar a los trabajadores y trabajadoras condiciones de seguridad y salud adecuadas en su ambiente de trabajo, lo cual conlleva al ejercicio pleno de sus facultades físicas $y$ mentales mediante la promoción del trabajo seguro y saludable, así como la prevención de los accidentes de trabajo y enfermedades ocupacionales.

Sin duda, esta Ley abarca lo concerniente a la promoción de la salud de los trabajadores, prevención de enfermedades profesionales, accidentes de trabajo, atención, rehabilitación, reinserción; determinando las prestaciones dinerarias que correspondan por los daños que ocasionen enfermedades ocupacionales $\mathrm{y}$ accidentes de trabajo, entre otros aspectos. A su vez, Landaeta (2010) expresa que los avances de estos últimos treinta años han influido en la salud de los trabajadores y las medidas para la disminución de los accidentes se han desarrollado aceptablemente, sin que esto 
implique la resolución de todos sus problemas, pero se ha evolucionado de manera trascendental, tal es el caso de la implantación del servicio de salud en el trabajo.

Ante este panorama, las instituciones universitarias se han dedicado a crear secciones o departamentos en materia de seguridad e higiene, para lograr condiciones de trabajo óptimas y preservar así la salud del trabajador, y en beneficio del patrono, quien puede lograr una mejor productividad, donde las condiciones de trabajo mínimas no han de verse como accesorias de un contrato de trabajo, sino como requisitos legales aplicables en toda situación en que surja el hecho social trabajo.

En este marco de discusiones, en el sector universitario público, los niveles gerenciales han visto los efectos económicos negativos cuando se dan desfavorablemente las condiciones del medio ambiente de trabajo, así como también, los conflictos laborales que pueden ocurrir en las mismas; mientras que el mejoramiento de ello se reconoce como una condición necesaria para mejorar la calidad, incrementar la productividad, reducir el ausentismo y la rotación. Es por ello, que resulta perentorio mencionar la toma de conciencia que se ha ido produciendo entre los interlocutores sociales acerca del valor que tiene la vida y la salud de los trabajadores.

En esta realidad están inmersas las universidades públicas de la Costa Oriental del Lago de Maracaibo, que ante los nuevos cambios se hace imprescindible que los espacios o ambientes de trabajo, garanticen el desarrollo físico y mental de la masa trabajadora, para que la función social de trabajar no sea una carga para sus ejecutantes, sino que, por el contrario, permita la utilización del tiempo libre y descanso en beneficio del trabajador, permitiéndole poder desarrollarse intelectualmente, y cada día poder regresar sanamente a sus hogares después de una agradable jornada laboral cumplida con los valores de seguridad establecidos en la LOPCYMAT (2005).

En este caso particular, la observación directa por parte del investigador, además de algunas entrevistas directas e informales al personal involucrado en el área objeto de estudio, evidencian que existen debilidades en materia de prevención de salud laboral. En este sentido, se muestran deficiencias de prevención de salud laboral porque muchas veces no cuentan con los recursos humanos, económicos y materiales o sencillamente porque no existe una cultura de prevención.

Lo antes expuesto, a criterio del investigador, puede deberse a que los propósitos planteados en la política de la gestión de Seguridad Industrial, Ambiente e Higiene Ocupacional de las instituciones a ser analizadas no han logrado compenetrar los principios e intenciones que rigen el comportamiento del personal; los requisitos exigidos por las funciones gerenciales no proporcionan al trabajador el alcance de sus metas; la incertidumbre en las condiciones de trabajo escasamente le proveen de seguridad en la ejecución de sus actividades; hay poca intervención de las autoridades universitarias en el proceso de integración de temas medioambientales, así como la falta de implicación de los trabajadores.

Lo señalado anteriormente, trae como consecuencias: incumplimiento de las metas, poco compromiso en los objetivos planteados y su internalización por las diferentes unidades o departamentos; el personal no se siente involucrado en la gestión de seguridad industrial, ambiente e 
higiene ocupacional, lo que genera descontrol en cierta medida del impacto de sus actividades en el ambiente interno; por último, los sistemas y registros quedan en un mero conjunto de documentos sin más trascendencia en su aplicación y prevención.

Bajo esta óptica, vale resaltar la conveniencia de realizar un análisis de las condiciones de trabajo de seguridad industrial, ambiente e higiene ocupacional en las universidades públicas de la Costa Oriental del Lago. Para ello, el autor considera importante abordar la variable objeto de estudio, a través de las condiciones de trabajo inseguro, incidente, enfermedad ocupacional, accidente de trabajo, riesgo laboral y orden - limpieza.

\section{Gestión de Seguridad Industrial, Ambiente e Higiene Ocupacional (SIAHO)}

Para la Norma Venezolana COVENIN 4001:2000 (2000), la gestión de seguridad industrial, ambiente e higiene ocupacional (SIAHO), es aquella que define la política de prevención y que incluye la estructura organizativa, responsabilidades, prácticas, procedimientos, procesos y recursos; por tanto, para llevar a cabo dicha política debe existir un sistema general de gestión, y éste debe ser parte integrante del mismo.

De acuerdo con la Cámara Petrolera (2011), la gestión de seguridad industrial, ambiente e higiene ocupacional (SIAHO), contempla herramientas con las cuales cuenta una empresa u organización para prevenir eventos no deseados, específicamente OHSAS 18001 (Occupational Health and Safety Assessment Series), es un estándar de gestión de prevención de riesgos laborales desarrollado por un consorcio internacional de entidades de normalización y de certificación. Esto tiene como propósito establecer los requisitos para un sistema de gestión en seguridad y salud ocupacional, que permita a una organización controlar sus riesgos de seguridad $\mathrm{y}$ salud ocupacional y mejorar su desempeño. Por su parte, la OHSAS 18001: 2007 (2007) aborda estos aspectos, pero no establece criterios ni detalla especificaciones para el diseño del sistema de gestión.

Para Bellido (2012), la gestión de seguridad industrial, ambiente e higiene ocupacional es aplicable a cualquier organización, pues permite establecer un SGSSO con el objeto de minimizar los riesgos para los empleados y otras partes interesadas. Permite implementar, mantener y mejorar continuamente un SGSSO; asegurar por si misma su conformidad con la política establecida en el SGSSO; demostrar tal conformidad a otros; buscar certificación; hacer una autodeterminación y declaración de conformidad de esta norma AA/1 3 .

En función de lo anteriormente expuesto, el sistema de gestión de seguridad y salud ocupacional OHSAS 18001:2007 (2007) especifica que muchas organizaciones gestionan sus operaciones por medio de la aplicación de un sistema de procesos y sus interacciones, el cual puede ser referido como el enfoque basado en procesos. Por otra parte, con la norma ISO 9001 se promueve el uso del enfoque de procesos. Dado que planear, hacer, verificar y actuar (PHVA) puede ser aplicado para todos los procesos, las dos metodologías se consideran compatibles.

Por consiguiente, un sistema de este tipo habilita a una organización para desarrollar su política de seguridad y salud ocupacional, establecer objetivos y 
procesos para el logro de los compromisos de la política, tomar acción cuando sea requerida para mejorar su desempeño y demostrar la conformidad del sistema en función de los requisitos de esta norma OHSAS (Occupational Health and Safety Assessment Series). El propósito global de la misma es apoyar y promover las buenas prácticas de $\mathrm{SSO}$, en balance con las necesidades socio-económicas.

Desde el enfoque de Quility Consulting Associate (2013), el sistema de gestión de seguridad y salud ocupacional de la norma OHSAS 18001:2007 (2007), reconocida internacionalmente, se aplica a cualquier organización tanto del sector de servicios como de manufactura. Su certificación demuestra su compromiso y decisión de mejorar las condiciones de seguridad y salud en el lugar de trabajo. Por otra parte, permite a la empresa mejorar su desempeño $\quad \mathrm{y} \quad$ distinguirse competitivamente en el mercado.

Aunado a lo expuesto en las líneas anteriores, es importante considerar lo señalado por Peralta (2010), quien refiere que la gestión de seguridad industrial, ambiente e higiene ocupacional se ocupa de proteger la salud de los trabajadores, controlando el entorno del trabajo para reducir o eliminar riesgos, con respecto al ambiente toma en cuenta los aspectos físicos que rodean al trabajador mientras desempeña su cargo, en relación a la higiene ocupacional se encarga de proteger y promover la salud y el bienestar de los trabajadores, salvaguardando la comunidad, así como el medio ambiente en general.

Por otra parte, Aguilar (2011) manifiesta que la gestión de seguridad industrial, ambiente e higiene ocupacional es la ciencia $\mathrm{y}$ arte dedicados al reconocimiento, evaluación y control de aquellos factores ambientales o tensiones emanados o provocados por el lugar de trabajo y que pueden ocasionar enfermedades, destruir la salud y el bienestar o crear algún malestar significativo entre los trabajadores o los ciudadanos de una comunidad.

De igual manera, Camisón et al. (2007) refieren que la gestión de seguridad industrial, ambiente e higiene ocupacional, es una herramienta de gestión que regula y normaliza el trabajo rutinario, así como repetitivo, que pone en valor el conocimiento de las circunstancias particulares de tiempo y lugar que poseen los operarios para la ejecución segura del trabajo.

Según la norma venezolana COVENIN 2260:2004 (2004), la seguridad industrial se define como a continuación se expresa.

Un conjunto de principios, leyes, criterios y normas formuladas, cuyo objeto fundamental es controlar el riesgo de accidentes que pudiesen derivar en lesiones a las personas, al igual que daños a los materiales $y$ equipos intervinientes en el desarrollo de toda actividad productiva (p. 2).

Mientras que en esta misma norma COVENIN 2260:2004 (2004), la higiene ocupacional se define como se transcribe seguidamente.

La ciencia dedicada al conocimiento, evaluación y control de aquellos factores ambientales o tensiones emanadas o provocadas por o con motivo del trabajo pudiendo ser causantes de enfermedades, afectar la salud y el bienestar, o crear algún malestar significativo entre los trabajadores o los ciudadanos de la comunidad (p. 1). 
Adicionalmente, Rodríguez (2010) profundiza sobre el tema de la seguridad e higiene industrial, señalando la necesidad de las organizaciones de la correcta ejecución de las actividades, dentro de un ambiente seguro, minimizando los riesgos que afecten la salud física y mental de sus trabajadores, capacitando al personal, definiendo mejor las actividades a desarrollar de acuerdo a sus capacidades, lo cual es posible a través del establecimiento de normas, las cuales deben desarrollarse considerando la presencia de los riesgos laborales, por tanto, el autor explica que el análisis de riesgos y accidentes constituye una actividad ligada a toda función administrativa, pues está en juego la salud del recurso humano.

En este compendio de ideas, el investigador considera la gestión de seguridad industrial, ambiente e higiene ocupacional como un sistema que implica especificaciones que deben cumplirse de acuerdo con un estándar establecido por un ente reconocido, orientado a sistemas de gestión de la salud y la seguridad en el trabajo, con la finalidad de garantizar ambientes de trabajo adecuados en las instituciones universitarias públicas de la Costa Oriental del Lago de Maracaibo. Ésta será medida a través de: condiciones de trabajo, elementos de la gestión de SIAHO, funciones de la gestión de SIAHO y factores que influyen en la gestión de SIAHO.

\section{Condiciones de trabajo}

De acuerdo con la Ley Orgánica de Prevención, Condiciones y Medio Ambiente de Trabajo (LOPCYMAT) (2005), Artículo 4, se entiende por condiciones de trabajo, a los efectos de esta Ley:
Las condiciones generales $\mathrm{y}$ especiales bajo las cuales se realiza la ejecución de las tareas. Los aspectos organizativos funcionales de las empresas y empleadores en general, los métodos, sistemas o procedimientos empleados en la ejecución de las tareas, los servicios sociales que éstos prestan a los trabajadores y los factores externos al medio ambiente de trabajo que tienen influencias sobre él.

Para Ruíz (2007), las condiciones de trabajo indican que la constante e innovadora mecanización del trabajo, los cambios de ritmo, de producción, los horarios, las tecnologías y aptitudes personales, entre otros, generan una serie de situaciones que pueden afectar a la salud, son las denominadas condiciones de trabajo, a las que se define como el conjunto de variables que definen la realización de una tarea en un entorno, determinando la salud del trabajador en función de tres variables: física, psicológica y social.

Desde el criterio de COVENIN 2273-91 (1991), las condiciones de trabajo o ambiente de trabajo se refieren al conjunto de elementos físicos, químicos, biológicos, sociales y culturales que rodean a una persona en el interior de su espacio de trabajo.

Tomando en cuenta los postulados descritos, para el investigador, las condiciones de trabajo están referidas a los elementos del lugar del trabajo, y la forma de realizar el trabajo. Cabe considerar el sector universitario público, en el cual juegan un papel importante las condiciones de trabajo, pues cualquier característica del trabajo realizado puede tener una 
influencia significativa en la generación de riesgos para la salud del personal en función de una tarea. En este estudio se analiza a través de condiciones de trabajo inseguro, incidente, enfermedad ocupacional, accidente de trabajo, riesgo laboral y orden - limpieza.

\section{Condiciones de trabajo inseguro}

Para la Organización Internacional del Trabajo (OIT) (2009), es toda actividad que por acción u omisión del trabajador conlleva la violación de un procedimiento, norma, reglamento o práctica segura establecida, tanto por el Estado como por la empresa, que puede producir incidente, accidente de trabajo, enfermedad ocupacional o fatiga personal. Según la Comisión Venezolana de Normas Industriales (COVENIN, 2248-87, 1987), la condición de trabajo inseguro puede definirse como a continuación se cita.

Es toda actividad que conlleva la violación de un procedimiento, norma, reglamento o práctica segura establecida, tanto por el estado como por la empresa. Es decir, toda acción u omisión que puede producir incidentes, accidentes, enfermedad profesional o fatiga del trabajador (p. 85).

La OIT (2009) señala que la condición de trabajo insegura amerita el uso de técnicas que permitan eliminar o reducir el riesgo de sufrir lesiones en forma individual o daños materiales en equipos, máquinas, herramientas y locales. Dentro de las condiciones generales de seguridad, las medidas generales de orden y aseo dentro del área de trabajo son de vital importancia.

En esta perspectiva, Zurisadai (2008) expone que las condiciones de trabajo inseguras se relacionan con las instalaciones, equipos de trabajo, maquinaria y herramientas que no están en condiciones de ser usados y de realizar el trabajo para el cual fueron diseñados o creados, que ponen en riesgo de sufrir un accidente a la o las personas que los ocupan.

Visto de esta forma, para el investigador, la condición de trabajo insegura se corresponde con una conducta o comportamiento de las personas que no cumplen un nivel requerido para considerarse normal o dentro de rangos de normalidad en el ámbito de la prevención de riesgos y seguridad del trabajo. Dentro de este marco, para el sector universitario público, este tipo de conductas aumentan la probabilidad de generar un accidente con sus consecuentes daños, pérdidas materiales y humanas. En ese aspecto, cualquier característica de las condiciones de trabajo inseguro puede tener una influencia significativa en la generación de riesgos.

\section{Incidente}

Para la OIT (2009), un incidente es todo suceso acaecido en el curso del trabajo o en relación con este, que tuvo el potencial de ser un accidente en el que hubo personas involucradas sin que sufrieran lesiones o se presentaran daños a la propiedad y/o pérdida en los procesos. Asimismo, Sura (2011, p. 4) plantea que "es un suceso repentino no deseado que ocurre por las mismas causas que se presentan los accidentes, sólo que por cuestiones del azar no desencadena lesiones en las personas, daños a la propiedad, al proceso $\mathrm{o}$ al ambiente".

Del mismo modo, Ruíz (2007, p. 47) denomina incidente "cualquier suceso no esperado ni deseado que no dando lugar a pérdidas de la salud o lesiones a las 
personas puede ocasionar daños a la propiedad, equipos, productos o al medio ambiente, pérdidas de producción $o$ aumento de las responsabilidades legales".

En función de lo señalado, a efectos de la presente investigación, los incidentes son parte de una alerta necesaria para ser atendida por las organizaciones. Es decir, es la oportunidad para identificar y controlar las causas básicas que lo generaron, antes de que ocurra un accidente. En el ámbito del sector universitario público se debe tener presente la importancia de la verdadera prevención en materia de SIAHO, logrando que se minimicen los incidentes, por ello se deben adoptar las recomendaciones que se generan de la investigación con la finalidad de controlarlos y aplicar medidas para la prevención de riesgos que afectan la salud del personal en cualquier área.

\section{Enfermedad ocupacional}

Para Sura (2011, p. 4), la enfermedad ocupacional es "todo estado patológico contraído o agravado con ocasión del trabajo". La ley concibe la enfermedad ocupacional en forma más amplia que el instrumento derogado, al considerar que no sólo una enfermedad será ocupacional cuando se contrae el estado patológico, sino también, cuando sea agrave. El Instituto Nacional de Prevención, Salud y Seguridad Laboral (INPSASEL) (2008), afirma que por enfermedad ocupacional se entiende lo que seguidamente se expone.

Son los estados patológicos contraídos o agravados con ocasión del trabajo o exposición al medio en el que la trabajadora $o$ el trabajador se encuentra obligado a trabajar, tales como los imputables a la acción de agentes físicos y mecánicos, condiciones disergonómicas, meteorológicas, agentes químicos, biológicos, factores psicosociales y emocionales, que se manifiesten por una lesión orgánica, trastornos enzimáticos o bioquímicos, trastornos funcionales o desequilibrio mental, temporales o permanentes. De acuerdo con lo consagrado en La ley Orgánica de Prevención, Condiciones y Medio Ambiente de Trabajo Gaceta Oficial $\mathrm{N}^{\circ}$ 38.236, del 26 de julio de 2005, en su artículo 70 (p. 9).

Tomando como fundamento la opinión de García (2007), debe entenderse enfermedad ocupacional como un daño para la salud de los trabajadores/as que se produce por la interacción de éstos con el entorno laboral cuando el trabajo se desarrolla en unas condiciones inadecuadas. En consecuencia, no todas las enfermedades de etiología laboral pueden ser consideradas como enfermedad ocupacional en sentido técnico-legal.

A su vez, para la Norma COVENIN 2270:2002 (2002), la enfermedad ocupacional se refiere a las afecciones agudas o crónicas causadas de una manera directa por el ejercicio de la profesión o labor que realiza el trabajador y que produce incapacidad. Visto de esta forma, la declaración de enfermedad ocupacional es responsabilidad del empleador 0 empleadora, quien deberá realizarla dentro de las primeras 24 horas siguientes al diagnóstico de la patología de presunto origen ocupacional, según la LOPCYMAT (2005).

De acuerdo a lo indicado, para el investigador, la enfermedad ocupacional es un padecimiento contraído con ocasión del trabajo o por exposición al ambiente en que el trabajador se encuentre obligado a trabajar; y el que pueda ser originado por la 
acción de agentes, condiciones o factores, que se manifiesten por un daño o trastorno de la salud, temporales o permanentes. Con respecto al sector universitario público, las enfermedades ocupacionales son responsabilidad de los empleadores, quienes deben tomar las medidas de seguridad y asimismo están obligados a hacer del conocimiento de los trabajadores, tanto los peligros específicos de accidentes a los cuales están expuestos, como las normas esenciales de prevención.

\section{Accidente de trabajo}

Ruíz (2007, p. 50) plantea que un accidente de trabajo "es toda lesión funcional $o$ corporal, permanente $o$ temporal, inmediata o posterior, o la muerte, resultante de una acción que pueda ser determinada o sobrevenida en el curso del trabajo, por el hecho o con ocasión del trabajo". Se incluyen los accidentes desde, hasta el lugar de trabajo y los que sucedan en ejercicio de actividad sindical.

En este sentido, la Norma COVENIN 2270:2002 (2002), señala como accidente de trabajo "el estado patológico con ocasión del trabajo, inmediata o posterior, o la muerte, resultante de la acción violenta de una fuerza exterior que pueda ser determinada o sobrevenida en el curso del trabajo por el hecho o con ocasión del trabajo". La LOPCYMAT, Artículo 69 (2005), define al accidente de trabajo como:

Todo suceso que produzca en el trabajador o la trabajadora una lesión funcional o corporal, permanente o temporal, inmediata o posterior, o la muerte, resultante de una acción que pueda ser determinada o sobrevenida en el curso del trabajo, por el hecho o con ocasión del trabajo.
Dentro de este orden de ideas planteadas, esta investigación se enfoca desde el punto de vista preventivo, estudiando sus causas, fuentes, agentes, tipos; generados en el sitio donde ejecuta su trabajo, todo ello con el fin de desarrollar la prevención. En el contexto del sector universitario público, evitar los accidentes de trabajo permitirá controlar los eventos, la recurrencia de los riesgos y garantizará el bienestar en las condiciones de trabajo.

\section{Riesgo laboral}

De acuerdo con Cabaleiro (2010), los riesgos laborales son aquellos que se producen por el hecho o en ocasión del trabajo a través de dos manifestaciones: los accidentes $\mathrm{y}$ las enfermedades profesionales, cuyos efectos pueden generar situaciones de invalidez temporaria o permanente, y cuyas consecuencias pueden variar entre la curación, la huella de alguna secuela, e inclusive la posibilidad de que la víctima muera.

En el caso de la Norma COVENIN 2270:2002 (2002), el riesgo laboral es la posibilidad de ocurrencia de un accidente de trabajo o de una enfermedad profesional. Al mismo tiempo Aguilera (2010), afirma que es como: la medida de la posibilidad y magnitud de los impactos adversos, siendo la consecuencia del peligro, y está en relación con la frecuencia con que se presente el evento.

Tomando en cuenta lo expuesto, a criterio del investigador, el riesgo laboral está presente al exponerse a una fuente de peligro en combinación con una actividad determinada donde probablemente ocurra un daño. Frente a esta formulación, en el un daño. Frente a esta formulación, en el sector universitario público en materia de SIAHO, estos riesgos representan que un 
trabajador sufra un determinado daño derivado del trabajo, valorándose la probabilidad de que ocurra y la severidad del mismo conjuntamente.

\section{Orden - limpieza}

Para Cortés (2010, p. 167), orden y limpieza, "es uno de los factores que más influencia ejercen en la prevención de accidentes, precisamente en el área de trabajo, ya que además de suprimirse con ello un elevado número de condiciones de inseguridad origen de múltiples accidentes". De esta manera, contribuye a la seguridad por el efecto psicológico que ejerce sobre la población trabajadora.

En el caso de la OIT (2009), el orden se entiende "la organización que permite disponer de un lugar adecuado para cada cosa y que cada cosa se mantenga en el lugar asignado para ello". El orden, comprende la señalización de los puestos de trabajo, pasillos o zonas de tránsito; el correcto almacenaje, control de materias primas y herramientas. Dicho de otro modo, Aguilera (2010) indica que la limpieza como complemento del orden, comprende la pintura adecuada de los techos, suelos, paredes; la retirada de chatarra, productos de desechos; la limpieza de suelos y ventanas, entre otros.

En concordancia con lo planteado, para el autor de esta investigación, la carencia del orden-limpieza, está relacionado con la falta de las más elementales normas de conservación en el lugar de trabajo, lo cual constituye una de las principales causas de los accidentes de trabajo. En ese aspecto, el sector universitario público, supone la organización de sus materiales, herramientas y equipos de tal manera que garantice un ambiente de trabajo donde el individuo pueda interactuar sin dificultad con su medio laboral.

\section{MÉTODO}

Metodológicamente se tipificó como descriptiva, con diseño de campo, no experimental y transeccional. La población estuvo constituida por las universidades públicas de la Costa Oriental del Lago: Universidad del Zulia en el Núcleo Costa Oriental del Lago, Universidad Nacional Experimental Rafael María Baralt y la Universidad Politécnica Territorial del Zulia, sede Cabimas, cuyas unidades informantes estuvieron compuestas por cuarenta y siete (47) trabajadores del área de mantenimiento y seis (6) supervisores del área de seguridad industrial, ambiente e higiene ocupacional, para un total de cincuenta y tres (53) personas.

Para la recolección de datos se utilizó la encuesta mediante la aplicación de un cuestionario, el mismo quedó conformado por dieciocho (18) ítems con escala de frecuencia. La validez se realizó a través del juicio de expertos, y para calcular su confiabilidad se empleó el método de Alfa de Cronbach, obteniéndose un valor de 0,96. El análisis de los datos se realizó mediante estadística descriptiva, con base en la media aritmética según el baremo mostrado en el cuadro 1. 
Cuadro 1. Baremo para la interpretación de la media aritmética

\begin{tabular}{lll}
\hline Opción de respuesta & Rango para la media & Nivel de respuesta para la variable \\
\hline Siempre(S) & $4,21-5,00$ & Muy alta presencia \\
Casi Siempre (CS) & $3,41-4,20$ & Alta presencia \\
Algunas Veces $(\mathrm{AV})$ & $2,61-3,40$ & Moderada presencia \\
Casi Nunca $(\mathrm{CN})$ & $1,81-2,60$ & Baja presencia \\
Nunca(N) & $1,00-1,80$ & Muy baja presencia \\
\hline
\end{tabular}

\section{RESULTADOS}

A efectos de iniciar el análisis, se presentan los resultados obtenidos para cada uno de los indicadores que conforman la dimensión condiciones de trabajo, sistematizado según el investigador en: condiciones de trabajo inseguro, incidente, enfermedad ocupacional, accidente de trabajo, riesgo laboral y orden-limpieza, dirigido a diagnosticar las condiciones de trabajo de seguridad industrial, ambiente e higiene ocupacional en las universidades públicas de la Costa Oriental del Lago.
La tabla 1 muestra el comportamiento de las respuestas suministradas por las unidades informantes en función del indicador condiciones de trabajo inseguro perteneciente a la dimensión condiciones de trabajo, arrojando una media de 3,55 para este indicador, implicando alta presencia del uso de técnicas que permitan eliminar o reducir el riesgo de sufrir lesiones en forma individual o daños materiales en equipos, máquinas, herramientas y locales.

Tabla 1. Condiciones de trabajo inseguro

En la universidad donde usted trabaja:

1 Se logra identificar toda actividad que ocasiona accidentes en el puesto de trabajo

2 Se utilizan técnicas de recolección de datos que permitan mostrar el riesgo de sufrir lesiones

en forma individual

3 Se cuenta con normativas de procedimientos para la ejecución de las tareas

\begin{tabular}{lllll}
\hline \multicolumn{1}{c}{$\begin{array}{c}\text { Promedio de las } \\
\text { respuestas } \\
\text { codificadas }\end{array}$} & \multicolumn{1}{c}{$\mathbf{1}$} & \multicolumn{3}{c}{ Ítems } \\
Categoría & 3,98 & 3,96 & & $\mathbf{2}$ \\
Promedio Indicador & Alta presencia & Alta presencia & Moderada presencia \\
Categoría & 3,55 & & \\
\hline
\end{tabular}

Atendiendo a los valores individuales de cada ítem, se tiene alta presencia al considerar los encuestados que se logra identificar toda actividad que ocasiona accidentes en el puesto de trabajo $(3,98)$ y se utilizan técnicas de recolección de datos que permitan mostrar el riesgo de sufrir lesiones en forma individual $(3,96)$; no obstante, con moderada presencia se cuenta con normativas de procedimientos para la ejecución de las tareas $(2,72)$. 
Al contrastar estos resultados con la teoría consultada, en universidades públicas de la Costa Oriental del Lago se evidencia que la seguridad industrial, ambiente e higiene ocupacional se gerencia atendiendo lo establecido por la Comisión Venezolana de Normas Industriales (COVENIN 2248-87, 1987), la cual expresa que toda acción u omisión puede producir incidentes, accidentes, enfermedad profesional o fatiga del trabajador.

Al respecto, es importante considerar que las condiciones de trabajo inseguras están relacionadas, tal como lo expone Zurisadai (2008), con las instalaciones, equipos de trabajo, maquinaria $\mathrm{y}$ herramientas que no están en condiciones de ser usados y de realizar el trabajo para el cual fueron diseñados o creados, que ponen en riesgo de sufrir un accidente a la o las personas que los ocupan.

Asimismo, existe congruencia con lo expuesto por el investigador al considerar que el sector universitario público, presenta condiciones de trabajo inseguras que aumentan la probabilidad de generar un accidente con sus consecuentes daños, pérdidas materiales $\mathrm{y}$ humanas, en tal sentido se gestiona para minimizar este tipo de riesgo laboral.

Seguidamente, en la tabla 2 se resume el comportamiento del indicador incidente, donde se observa para este indicador una media de 4,04 que implica alta presencia al momento de identificar y controlar las causas básicas de que ocurra un accidente. Adicional a esto, se evidencian medias de 3,72; 3,72 y 4,68 para los ítems 4; 5 y 6 respectivamente, ubicándolos en la categoría de alta presencia, específicamente al referirse que el trabajador logra identificar situaciones que tengan la probabilidad de causar accidentes, así como situaciones no deseadas que puedan causar lesiones, además de que se atienden con prontitud los incidentes presentados en el área de trabajo.

Tabla 2. Indicador: Incidente

En la universidad donde usted trabaja:

4 El trabajador logra identificar situaciones que tengan la probabilidad de causar accidente

5 El trabajador identifica situaciones no deseadas que puedan causar lesiones

6 Se atienden con prontitud los incidentes presentados en el área de trabajo

\begin{tabular}{lllll}
\hline \multicolumn{1}{c}{$\begin{array}{c}\text { Promedio de las } \\
\text { respuestas } \\
\text { codificadas }\end{array}$} & \multicolumn{1}{c}{$\mathbf{4}$} & \multicolumn{3}{c}{ Ítems } \\
Categoría & 3,72 & 3,72 & $\mathbf{5}$ & 4,68 \\
Promedio Indicador & Alta presencia & Alta presencia & Muy alta presencia \\
Categoría & 4,04 & & \\
\hline
\end{tabular}


En consideración a estos resultados, en las instituciones bajo estudio, con alta presencia se gerencia la seguridad industrial, ambiente e higiene ocupacional, de manera que se logra prevenir cualquier suceso no esperado ni deseado que dando lugar a pérdidas de la salud o lesiones a las personas puede ocasionar daños a la propiedad, equipos, productos o al medio ambiente, pérdidas de producción o aumento de las responsabilidades legales, tal como lo expresa Ruíz (2007).

Los valores alcanzados para el indicador también validan lo expuesto por el investigador, al considerar que el ámbito del sector universitario público debe tener presente la importancia de la verdadera prevención en materia de SIAHO, logrando que se minimicen los incidentes.

En lo que respecta al indicador enfermedad ocupacional, se tiene una media de 2,91 que indica moderada presencia del mismo, lo cual está asociado a las condiciones de trabajo de seguridad industrial, ambiente e higiene ocupacional en las instituciones universitarias analizadas, tal como se muestra en la tabla 3. Asimismo, se observa, de acuerdo al comportamiento de las medias de los ítems, que en todas las actividades relacionadas se alcanza la categoría de moderada presencia.

Tabla 3. Indicador: Enfermedad ocupacional

En la universidad donde usted trabaja:

7 Se proporcionan condiciones ergonómicas para que el trabajador ejecute su trabajo

8 El trabajador está expuesto a factores de riesgo debido a los productos empleados

9 El trabajador está expuesto a un ambiente inseguro

\begin{tabular}{|c|c|c|c|}
\hline \multirow{3}{*}{$\begin{array}{l}\text { Promedio de las } \\
\text { respuestas codificadas }\end{array}$} & \multicolumn{3}{|c|}{ Ítems } \\
\hline & 7 & 8 & 9 \\
\hline & 2,72 & 3,06 & 2,96 \\
\hline Categoría & $\begin{array}{l}\text { Moderada } \\
\text { presencia }\end{array}$ & Moderada presencia & Moderada presencia \\
\hline Promedio Indicador & 2,91 & & \\
\hline Categoría & Moderada presencia & & \\
\hline
\end{tabular}

Así, en las universidades bajo estudio, la enfermedad ocupacional se caracteriza, en moderada presencia, porque se proporcionan condiciones ergonómicas para que el trabajador ejecute su trabajo $(2,72)$, el trabajador está expuesto a factores de riesgo debido a los productos empleados $(3,06)$, así como a un ambiente inseguro $(2,96)$.
La situación mostrada, pone en evidencia que las universidades bajo estudio, tratan de gerenciar la seguridad industrial, ambiente e higiene ocupacional, propiciando condiciones adecuadas para el desarrollo del trabajo, buscando con ello evitar cualquier enfermedad ocupacional de los trabajadores/as (García, 2007). A este respecto, para la Norma COVENIN 
2270:2002 (2002), la enfermedad ocupacional comprende las afecciones agudas o crónicas causadas de una manera directa por el ejercicio de la profesión o labor que realiza el trabajador y que produce incapacidad.

Con base a lo descrito, también se valida la posición del investigador, al referir que el sector universitario público evidencia enfermedades ocupacionales, las cuales son responsabilidad de los empleadores, quienes deben tomar las medidas de seguridad y asimismo están obligados a hacer del conocimiento de los trabajadores, tanto los peligros específicos de accidentes a los cuales están expuestos, como las normas esenciales de prevención.
En la tabla 4 se resume el comportamiento del indicador accidente de trabajo, donde se observa que el mismo alcanzó un promedio de 3,93 que indica alta presencia del indicador. De manera específica, puede observarse como para los ítems 10; 11 y 12 alcanzan una alta presencia, pues los encuestados consideran que se proporcionan condiciones de trabajo para evitar al trabajador cualquier lesión permanente $(3,72)$; se utilizan registros de los eventos que pueden producir una lesión en el trabajador $(4,04)$ y se identifica con facilidad un accidente de trabajo $(4,04)$.

Tabla 4. Accidente de trabajo

En la universidad donde usted trabaja:

10 Se proporcionan condiciones de trabajo para evitar al trabajador cualquier lesión permanente

11 Se utilizan registros de los eventos que pueden producir una lesión en el trabajador

12 Se identifica con facilidad un accidente de trabajo

\begin{tabular}{llll}
\hline \multicolumn{1}{c}{$\begin{array}{c}\text { Promedio de las } \\
\text { respuestas codificadas }\end{array}$} & \multicolumn{1}{c}{$\mathbf{1 0}$} & $\mathbf{1 1}$ & $\mathbf{1 2}$ \\
& 3,72 & 4,04 & 4,04 \\
Categoría & Alta presencia & Alta presencia & Alta presencia \\
Promedio Indicador & 3,93 & & \\
Categoría & Alta presencia & & \\
\hline
\end{tabular}

Con base a lo descrito, puede decirse entonces que las universidades públicas de la Costa Oriental del Lago implementan una gerencia enfocada en la seguridad industrial, ambiente e higiene ocupacional, de manera tal que buscan evitar cualquier suceso que produzca en el trabajador (a) alguna lesión o la muerte, resultante de una acción que pueda ser determinada o sobrevenida en el curso del trabajo, por el hecho o con ocasión del trabajo, definida por la LOPCYMAT en su artículo 69 (2005) como accidente de trabajo. 
Situación que coincide con el criterio del investigador, al considerar que evitar los accidentes de trabajo le permitirá controlar los eventos, la recurrencia de los riesgos y garantizará el bienestar de las condiciones de trabajo en las instituciones universitarias públicas de la Costa Oriental del Lago.

Ahora bien, en el orden de presentación de los resultados, se tienen los valores para el indicador riesgo laboral. En este sentido, en la tabla 5 se observa un promedio de 2,47 indicando baja presencia según las respuestas dadas por los encuestados. Al desglosar los resultados por ítems, se evidencia baja presencia de que se instruye al personal a través de actividades de gestión del SIAHO, la identificación de ocurrencia de un accidente de trabajo (2,34); de utilizar instrumentos que permiten medir la frecuencia del peligro en el área de trabajo $(2,36)$ y de contar con normas que regulan el riesgo laboral $(2,70)$.

Tabla 5. Indicador: Riesgo laboral

En la universidad donde usted trabaja:

13 Se instruye al personal a través de actividades de gestión del SIAHO, la identificación de ocurrencia de un accidente de trabajo

14 Se utilizan instrumentos que permiten medir la frecuencia del peligro en el área de trabajo

15 Se cuenta con normas que regulan el riesgo laboral

\begin{tabular}{llll}
\hline \multicolumn{1}{c}{$\begin{array}{c}\text { Promedio de las } \\
\text { respuestas codificadas }\end{array}$} & \multicolumn{1}{c}{$\mathbf{c}$} & \multicolumn{1}{c}{$\mathbf{1 5}$} \\
& \multicolumn{1}{c}{$\mathbf{1 3}$} & & 2,36 \\
Categoría & 2,34 & Baja presencia & Moderada presencia \\
Promedio Indicador & Baja presencia & & \\
Categoría & 2,47 & & \\
\hline
\end{tabular}

Estos resultados ponen en evidencia que las universidades bajo estudio, al gerenciar la seguridad industrial, ambiente e higiene ocupacional, con baja presencia buscan disminuir la posibilidad de ocurrencia de un accidente de trabajo o de una enfermedad profesional, expresada según la Norma COVENIN 2270:2002 (2002) como riesgo laboral.
Los valores alcanzados para el indicador también validan lo expuesto por el investigador, al considerar que en el ámbito del sector universitario público se debe valorar la probabilidad de que ocurra un accidente de trabajo o de una enfermedad profesional y la severidad del mismo conjuntamente. 
En cuanto al indicador orden - limpieza, los resultados revelan un promedio de 4,04 ubicándola en la categoría de alta presencia, tal como puede observarse en la tabla 6. En este sentido, los sujetos informantes afirman con alta presencia que se utiliza señalización en las áreas de tránsito $(3,72)$ y se mantiene el área de trabajo ordenada $(3,72)$; mientras que con muy alta presencia el trabajador dispone de un lugar adecuado para ordenar cada material o recurso de trabajo $(4,68)$.

Tabla 6. Orden - limpieza

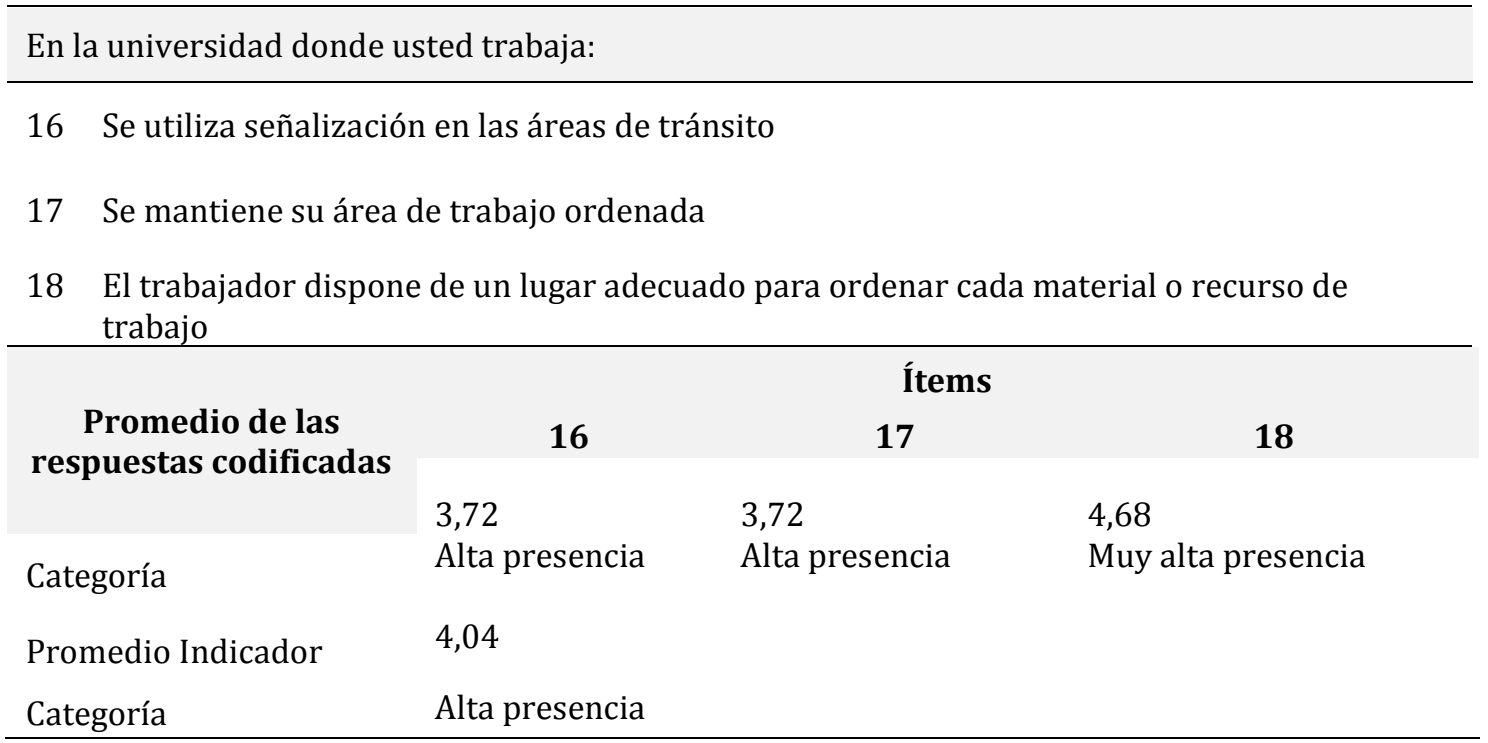

A partir de estos resultados, se encuentra congruencia con lo expuesto por Cortés (2010), quien refiere que el orden y limpieza constituye uno de los factores con más influencia en la prevención de accidentes, precisamente en el área de trabajo, ya que permite suprimir un elevado número de condiciones de inseguridad origen de múltiples accidentes.

De igual manera, alcanzan en gran medida a validar lo establecido por el investigador, cuando afirma que el sector universitario público, supone la organización de sus materiales, herramientas y equipos de tal manera que garantice un ambiente de trabajo donde el individuo pueda interactuar sin dificultad con su medio laboral.

Desde el punto de vista de la dimensión condiciones de trabajo, tal como se evidencia en la tabla 7, el valor promedio obtenido de sus indicadores fue de 3,49, ubicándose en el rango que va desde 3,41 hasta 4,20 con categoría de alta presencia, ello demostró que en las universidades públicas de la Costa Oriental del Lago de Maracaibo existe una alta presencia de las condiciones generales $y$ especiales bajo las cuales se realiza la ejecución de las tareas. Por ello, se deduce que hay alta congruencia con lo expuesto por la norma COVENIN 2273-91 (1991), la cual establece que las condiciones de trabajo o ambiente de trabajo, es el conjunto de elementos físicos, químicos, biológicos, sociales y culturales que rodean a una persona en el interior de su espacio de trabajo y permiten un entorno acorde, determinando la salud del trabajador. 
Tabla 7. Dimensión: Condiciones de trabajo

\begin{tabular}{lcl}
\hline Indicador & Media & Categoría \\
\hline Condiciones de trabajo inseguro & 3,55 & Alta presencia \\
Incidente & 4,04 & Alta presencia \\
Enfermedad ocupacional & 2,91 & Moderada presencia \\
Accidente de trabajo & 3,93 & Alta presencia \\
Riesgo laboral & 2,47 & Baja presencia \\
Orden - limpieza & 4,04 & Alta presencia \\
Promedio & $\mathbf{3 , 4 9}$ & Alta presencia \\
\hline
\end{tabular}

Visto así, también validan al investigador cuando afirma que las condiciones de trabajo en el sector universitario público, juega un papel importante, pues cualquier característica del trabajo realizado puede tener una influencia significativa en la generación de riesgos para la salud del personal en función de una tarea y es analizado con alta presencia en sus condiciones de trabajo inseguro $(3,55)$, incidente $(4,04)$, accidente de trabajo $(3,93)$, orden - limpieza $(4,04)$, con moderada presencia la enfermedad ocupacional $(2,91)$ y con baja presencia el riesgo laboral $(2,47)$.

\section{CONCLUSIONES}

A través de esta investigación se trató de dar respuesta al objetivo establecido; sobre la base de los resultados obtenidos, al análisis y discusión de los resultados producto de la aplicación del instrumento de recolección de datos. Siendo así como se formulan las siguientes conclusiones:

En relación al objetivo que estuvo orientado a identificar las condiciones de trabajo de seguridad industrial, ambiente e higiene ocupacional en las universidades públicas de la Costa Oriental del Lago de Maracaibo, se concluye que existe una alta presencia de las condiciones generales y especiales bajo las cuales se realiza la ejecución de las tareas.

Específicamente, se obtuvo que la seguridad industrial, ambiente e higiene ocupacional se gerencia atendiendo lo establecido por la Comisión Venezolana de Normas Industriales (COVENIN 2248-87, 1987); de igual manera se logra prevenir cualquier suceso no esperado ni deseado.

Asimismo, las universidades bajo estudio, tratan de gerenciar la seguridad industrial, ambiente e higiene ocupacional, propiciando condiciones adecuadas para el desarrollo del trabajo, buscando con ello evitar cualquier enfermedad ocupacional como un daño para la salud de los trabajadores/as, así como cualquier suceso que produzca en el (la) trabajador (a) alguna lesión o la muerte.

Al mismo tiempo, se evidenció que las universidades analizadas, al gerenciar la seguridad industrial, ambiente e higiene ocupacional, con baja presencia buscan disminuir la posibilidad de ocurrencia de un accidente de trabajo o de una enfermedad profesional, y el orden y limpieza, 
constituye uno de los factores con más influencia en la prevención de accidentes.

\section{REFERENCIAS}

Aguilar, E. (2011). Desarrollo de un modelo de gestión de seguridad e higiene industrial. Primera edición. España: Editorial EAE

Aguilera, J. (2010). Sistemas de gestión de la seguridad $y$ salud en el trabajo. Directivas de la OIT vs. Normas OHSAS 18001. Prevención, Trabajo y Salud

Bárbaro, L. (2010). Sistema de administración de la seguridad y salud en el trabajo. Colombia: Editorial Edue

Bellido, 0. (2012). Gestión de seguridad y salud ocupacional. Recuperado de http://www.slideshare.net/FIREHACK/ gestion-deseguridad-y-saludocupacional-presentation

Blanco, L. (2004). Accidentes laborales. Colombia: Editorial Edue

Cabaleiro, V. (2010). Prevención de riesgos laborales. Módulo transversal. España: Editorial Ideas propias, S.L

Cámara Petrolera (2011). Sistema de Gestión de seguridad y salud ocupacional de acuerdo a lo establecido por OHSAS 18001. Recuperado de http://www.cpzulia.org/ARCHIVOS_SS A/9nas_Jornadas_SHA_2007/23_05_07/ ohsas_18001_gyrodata.pdf

Camisón, C., Cruz, S. y González, T. (2007). Gestión de la Calidad: Conceptos, enfoques, modelos y sistemas. Madrid: Ediciones Pearson Educación, S.A

Comunidad Andina de Naciones (2005). Informe de la II reunión del comité andino de autoridades en seguridad y salud en el trabajo. Recuperado de http://www.ilo.org/wcmsp5/groups/p ublic/---ed_protect/---protrav/--safework/documents/policy/wcms_21 2074.pdf

Cortés, D. (2010). La seguridad en el trabajo. Madrid: Ediciones Pirámide

Fernández, R. (2005). Irlanda y Finlandia: dos modelos de especialización en tecnologías avanzadas. Policy Papers, $\mathrm{N}^{\circ}$
3. Madrid: Instituto Complutense de Estudios Internacionales

García, P. (2010). Salud laboral. México: Editorial Mason

García, E. (2007). Estrategias de responsabilidad social y gestión en seguridad industrial en el trabajo. Revista Cubana de Salud y Trabajo, 1(3), 51-69

Instituto Nacional de Prevención, Salud y Seguridad Laboral (INPSASEL) (2008). Recuperado

de http://www.inpsasel.gob.ve/moo_news /Prensa_409.html

Ley Orgánica de Prevención, Condiciones y Medio Ambiente de Trabajo (LOPCYMAT) (2005). Disposiciones Fundamentales. Recuperado de http://www.inpsasel.gob.ve/moo_news /lopcymat.htm

Norma Venezolana COVENIN 4001 (2000). Sistema de Gestión de Seguridad e Higiene Ocupacional. Requisitos. Recuperado de http://www.cpzulia.org/ARCHIVOS_SS A/4001_2000_SGSHO.pdf

Norma Venezolana COVENIN 2260 (2004). Sistema de Gestión de Seguridad e Higiene Ocupacional. Requisitos. Recuperado de http://www.sencamer.gob.ve/sencame r/normas/2260-04.pdf

Norma Venezolana COVENIN 2270 (2002). Comité de higiene y seguridad industrial en el trabajo. Guía para su integración y fundamento. Caracas: Editorial Fondonorma.

Norma Venezolana COVENIN 2248 (1987). Manejo de materiales y Equipos. Medidas generales de seguridad. Recuperado de http://www.sencamer.gob.ve/sencame r/normas/2248-87.pdf

Norma Venezolana COVENIN 2273 (1991). Principios ergonómicos de la concepción de los sistemas de trabajo. Recuperado de http://www.cpzulia.org/ARCHIVOS_SS A/2273_1991_principios_ergonomicos. pdf 
OHSAS 18001:2007 (2007). Requisitos. Sistema de Gestión en Seguridad y Salud Ocupacional. Recuperado de https://manipulaciondealimentos.files. wordpress.com/2010/11/ohsas18001-2007.pdf.

Organización Internacional del Trabajo (OIT). (2005). La seguridad en cifras. Estrategia global en materia de seguridad y salud en el trabajo. Conclusiones adoptadas por la Conferencia Internacional del Trabajo en su 91a reunión. Ginebra

Organización Internacional del Trabajo (OIT). (2009). Recomendaciones técnicas (OIT). Oficina Internacional del Trabajo. Ginebra

Organización Mundial y Panamericana de la Salud (OMPS) (2005). Situación de Salud en las Américas: Indicadores Básicos. Recuperado de http://www.paho.org/hq/index.php?op tion $=$ com_content $\&$ view $=$ article\&id $=24$ 70\%3A2010-datastatistics\&catid=1900\%3Adatastatistics-hom

Peralta, U. (2010). Seguridad e higiene industrial. México: Editorial Mc Graw Hill
Quility Consulting Associate (2013). Gestión de Seguridad y Salud Ocupacional OHSAS 18001. Recuperado de http://www.qcaquality.com.ar/gestionde-seguridad-y-salud-ocupacionalohsas.html

Rodríguez, J. (2010). Administración de pequeñas y medianas empresas. 6ta. Edición. México: Cengage Learning Editores

Ruíz, G. (2007). Seguridad industrial. México: Editorial Limusa

Sura, K. (2011). Política de seguridad y liderazgo. Madrid: Editorial Prentice Hall

VanDer, R. (2010). La higiene ocupacional en América Latina. Washington, D.C.: Organización Panamericana de la Salud

Zúñiga, L. (2005). Reducción de los accidentes del trabajo mediante el cambio de la conducta hacia la seguridad. Revista Mapfre Seguridad. España

Zurisadai, J. (2008). Análisis y gestión de la productividad. Buenos Aires: Editorial Osmar 